\title{
Effects of pomegranate juice on cardiometabolic risk factors and biomarkers of oxidative stress and inflammation in hemodialysis patients, a crossover controlled trial
}

\section{Reza Barati Boldaji}

Shiraz University of Medical Sciences

Masoumeh Akhlaghi ( $\nabla$ msm.akhlaghi@gmail.com )

Shiraz University of Medical Sciences https://orcid.org/0000-0003-3868-0227

Mohammad Mehdi Sagheb

Shiraz University of Medical Sciences

Zahra Esmaeilinezhad

Shiraz University of Medical Sciences

\section{Research Article}

Keywords: Pomegranate, hemodialysis, hypertension, oxidative stress, inflammation, lipid profile

Posted Date: January 15th, 2019

DOI: https://doi.org/10.21203/rs.2.220/v1

License: (c) (1) This work is licensed under a Creative Commons Attribution 4.0 International License.

Read Full License 


\section{Abstract}

Background: Pomegranate has antioxidant, cardioprotective, and anti-inflammatory properties. We questioned if pomegranate juice (PJ) benefits lipid profile and oxidative and inflammatory biomarkers of hemodialysis patients.

Methods: The study had a crossover design. Hemodialysis patients $(n=41)$ were divided into two groups: PJ-treated group who received $100 \mathrm{ml}$ natural PJ immediately after their dialysis session three times a week and the control group who received the usual care. After 8 weeks, a 4-week washout period was established and afterwards the role of the groups was exchanged. Lipid profile, blood pressure, and oxidative and inflammatory biomarkers were measured before and after each sequence. Comparisons between the two conditions were performed by ANCOVA with adjustments for potential confounders.

Results: Based on results of intention-to-treat analysis, triglycerides were decreased in PJ condition and increased in the control. Conversely, high-density lipoprotein (HDL)-cholesterol was increased in PJ and decreased in control. There was a significant between-condition difference for both triglycerides and HDLcholesterol $(P<0.001)$. Total and low-density lipoprotein $(L D L)$-cholesterol did not significantly change in either condition. Systolic and diastolic blood pressure significantly decreased in PJ condition and caused a significant difference between two conditions $(P<0.001)$. Total antioxidant capacity increased in PJ condition $(P<0.001)$ and decreased in control $(P<0.001)$. Conversely, malondialdehyde and interleukin- 6 decreased in $P J(P<0.001)$ and increased in the control $(P \leq 0.001)$. The changes of these biomarkers were significantly different between two conditions.

Conclusions: In conclusion, 8-week PJ consumption showed beneficial effects on blood pressure, serum triglycerides, HDL cholesterol, oxidative stress, and inflammation in hemodialysis patients. The trial was registered at Iranian Registry of Clinical Trials (ID number: IRCT 2016070428797N1).

Keywords: Pomegranate, hemodialysis, hypertension, oxidative stress, inflammation, lipid profile.

\section{Background}

Chronic kidney disease (CKD) is a long-term kidney disease characterized by gradual loss of kidney function [1]. End-stage renal disease (ESRD) occurs in the last stages of CKD, when kidneys lose most of their function in filtering blood from wastes [1]. Although accumulation of fluid, toxins, and wastes in the body is the primary dilemma in ESRD, the patients are implicated in comorbidities such as cardiovascular diseases [2,3], mainly as a result of blood lipid disorders [1,4], hypertension [5], oxidative stress [3], and inflammation [6].

Lipid profile abnormalities in CKD generally include elevated triglycerides and reduced levels of highdensity lipoprotein (HDL) cholesterol with either no remarkable change or a reduction in low-density lipoprotein (LDL) cholesterol [7]. This is especially important because compared to total and LDL cholesterol, low HDL cholesterol and elevated triglyceride concentrations predict more strongly 
cardiovascular disease [7]. These lipid abnormalities are likely due to post-translational modification of apolipoproteins, especially B100, by glycation, oxidation, and carbamylation (the last one resulted from spontaneous decomposition of urea) which leads to prolonged delipidation and thus appearance of small-dense LDL particles and highly atherogenic chylomicron and very low-density lipoprotein remnants [7].

High blood pressure is both a trigger and a consequence of CKD [8]. High blood pressure in CKD may be the result of fluid retention, altered sodium homeostasis, and consequently activation of reninangiotensin-aldosterone system [8]. Also, oxidative stress which comes along with CKD may promote endothelial dysfunction through suppression of nitric oxide bioavailability.

CKD is also a case of oxidative stress and inflammation. Oxidative stress may be considered as a tool for prediction of morbidity and mortality in CKD patients $[9,10]$. CKD patients have low levels of antioxidants, such as vitamin $\mathrm{C}$ and glutathione, and high concentration of pro-oxidants because of increased production of reactive oxygen and nitrogen species by a variety of means, for instance, damaged nephrons, uremic toxins, and bio-incompatibility of dialysis membranes and solutions $[11,12]$. On the other hand, oxidative stress and inflammation go hand in hand; it is not only oxidative stress that acts as a trigger for inflammatory response but inflammation in turn recruits phagocytes into the injured region, leading to production of oxygen radicals [13].

Hemodialysis is the last strategy for the treatment and survival of ESRD patients when they are on waiting list for kidney transplantation. While the procedure attenuates blood pressure, it has no effect on lipid profile, but also exacerbates severity of oxidative stress and inflammation [12]. Thus, consumption of foods with broad biological advantageous may be beneficial for ESRD patients. Pomegranate (Punica granatum L.) is an ancient fruit, cultivated all around the world. Due to extensive health benefits, pomegranate has been the focus of many in vitro, animal, and human studies [14]. The beneficial effects of pomegranate are attributed to polyphenols which are phytochemicals with great antioxidant, anticarcinogenic, anti-diabetic, cardioprotective, and anti-inflammatory capabilities [15].

Very few available studies on the effect of pomegranate in hemodialysis patients found conflicting results for cardiovascular risk factors and no effect for markers of oxidative stress and inflammation [1618]. The controversy or negative results may be due to the time of pomegranate administration which has been mostly before dialysis session when polyphenols is quickly excreted during dialysis. Therefore, in the current study we examined the effect of postdialysis administration of pomegranate juice (PJ) on lipid profile and biomarkers of oxidative stress and inflammation in hemodialysis patients.

\section{Methods}

\section{Study design}


The trial was preformed according to a crossover design in spring and summer 2016. Hemodialysis patients were selected from dialysis centers in Namazi and Shahid Faghihi hospitals of Shiraz, Iran. A sample size of 19 was determined based on results provided by previous investigators [19], a type 1 error of $5 \%$, power of $20 \%$, and considering a $10 \%$ drop-out rate.

Inclusion criteria were as follows: ESRD patients on dialysis treatment, dialysis 3 times a week for at least 3 months, an age range of 18 to 65 years, life expectancy more than 1 year, serum potassium level of less than $6 \mathrm{mEq} / \mathrm{L}$, and permission of the physician in charge of the patient. Patients were not included if they were involved in serious diseases such as malignancy, other organs' failure, taking antioxidant supplements such as vitamin E and vitamin C over the month before the study, iron sucrose (Venofer) usage at least two weeks before the study, malnutrition that required nutritional support, and hospitalization for $>5$ days per month. Our primary protocol was to exclude the patients if they lost inclusion criteria, missed PJ consumption for $>3$ days a month, changed hypertension and hyperlipidemia medications, did not follow dietary recommendations, and hospitalized during the intervention. However, none of the patients was excluded according to these criteria.

The study was approved by the Ethics Committee of Shiraz University of Medical Sciences (approval number. IR.SUMS.REC.1394.210), and registered at Iranian Registry of Clinical Trials (ID number: IRCT 2016070428797N1). Study protocol was explained and written informed consent was obtained from all participants.

\section{Intervention}

The study was performed in a crossover design with two 8-week sequences separated by a 4-week washout period. At the beginning, the participants were divided into two groups: PJ-treated group $(n=22)$ who consumed $100 \mathrm{~g}$ natural PJ after their dialysis session three times a week and the control group $(\mathrm{n}=$ 19) who received the usual care. After the 8-week intervention, there was a 4-week washout course and afterwards the second sequence of the study was started in which the role of the groups was interchanged. The Ethical Committee of our institution did not permit having a control treatment because 1) hemodialysis patients have restrictions for consumption of liquids; 2 ) it may not be ethically approved to ask hemodialysis patients to drink a placebo beverage when a beneficial effect for which could not be substantiated; 3 ) making a control drink for PJ required addition of artificial colors and pomegranate flavor which could be harmful for ESRD patients; and 4) in contrast to similar studies on hemodialysis patients, we administered PJ after dialysis session, thus it was crucial to avoid administering unnecessary food items. Because hemodialysis patients spend hours in the dialysis unit, to prevent contamination between intervention and control groups, we recruited the participants from two hospitals and allocated each of the intervention and control groups to a hospital at a time.

Pomegranate was from Rabab variety cultivated in Neyriz, Shiraz. Pomegranates were purchased from Neyriz pomegranate farms in autumn and stored at $-20 \otimes C$ until the time of the intervention. During the intervention, each week some pomegranates were thawed, crushed, and squeezed, and the juice was collected in $500 \mathrm{ml}$ bottles. Thus, the juice was prepared fresh and distributed amongst the patients in PJ 
condition every week. The patients were asked to keep the juice in a refrigerator until the time of use. One hundred milliliters measuring cups were provided for the participants and they were asked to drink $100 \mathrm{ml}$ PJ three times a week after their dialysis session. In order to ensure consumption of entire amount of PJ, each week $100 \mathrm{ml}$ extra PJ was given to the patients just in case if other family members wanted to taste it.

During the study, each patient was under supervision and care of a nephrologist and medical care team. Blood pressure was measured before and after PJ consumption in order to ensure that PJ would not cause hypotension. For all of the patients, weight maintenance diets were prescribed and the amount of fruit and vegetables that each patient could consume was explained. Diets were set in such a way that people in both groups received equal amounts of fruit and fruit juice. A list of high-potassium fruits was given to the patients and they were asked to abstain from high-potassium fruits on days of PJ consumption. The patients were also recommended to exclude pomegranate and PJ consumption throughout the intervention except for the PJ which was administered.

\section{Measurements}

Total antioxidant capacity and malondialdehyde were considered as the primary and interleukin- 6 was measured as the secondary outcome. All parameters were measured at baseline, the end of the first sequence, and before and after the second sequence of the intervention. For weight and body composition, measurements were performed immediately after the dialysis session [20]. Weight was measured by a digital scale (AM-2018) and body composition was determined by bioelectric impedance analyzer (BIA) (InBody, Biospace Co., South Korea). Blood pressure was measured in predialysis state twice with at least 5 min interval by a digital barometer (Microlife BP A200 AFIB(. The mean of two measurements was considered as the subject's blood pressure.

Blood samples were collected after 12-h fasting. All biochemical variables were measured in serum. Serum lipid profile was analyzed by an auto-analyzer (BT 1500, Biotecnica Instruments, Italy) using specific kits (Pars-Azmun, Iran). Malondialdehyde (MDA) was quantitated by thiobarbituric acid reactive substances (TBARS) method [21]. The method principle is based on the reaction of lipid peroxidation products in serum samples with thiobarbituric acid in the presence of trichloroacetic acid, butylated hydroxyltoluene, hydrochloric acid, and sodium dodecyl sulfate. The reaction mixture was then heated for $30 \mathrm{~min}$ at $95^{\circ} \mathrm{C}$ and the formed chromogen is read at $532 \mathrm{~nm}$. Interleukin-6 (IL-6) (Diaclone, France) and serum total antioxidant capacity (TAC) (Biocore diagnostics, Hamburg, Germany) were determined by colorimetric assay using commercially available kits.

Three-day dietary recall (two weekdays and one weekend day) was used to assess dietary intake and monitor dietary compliance. Dietary intakes were analyzed by Nutritionist IV software (First Databank, San Bruno, CA, USA) modified for Iranian foods. Mini nutritional assessment (MNA) was used as a tool for detecting malnutrition in hospitalized young and middle-aged adults [22].

\section{Statistical analyses}


Data were analyzed using SPSS software (version 22; IBM, Armonk, USA). Normality of data distribution was checked by visual examination of the histogram curve of data frequencies. In the case of abnormality, log-transformed data was used for determination of statistical difference between treatment conditions. The intention-to-treat (ITT) approach was used for the analysis. Comparisons between the two conditions were performed by ANCOVA (treatment $\times$ time interaction) with age and gender as the covariates. $P$ value $<0.05$ was considered statistically significant.

\section{Results}

After assessing 182 individuals, 57 patients who met the described inclusion criteria were found and 41 patients accepted to participate in the study. The patients were assigned to PJ $(n=22)$ and control $(n=19)$ groups for the first sequence. Due to stomach discomfort, one patient from PJ group could not continue PJ intervention and was excluded. In the second sequence, one person from the PJ group deceased and another moved away. Therefore, 38 and 40 patients completed the 8-week PJ and control conditions, respectively (Figure 1). However, the analysis was performed based on ITT approach, with 41 and 40 patients being in the PJ and control conditions, respectively. Table 1 indicates baseline characteristics of participants. The age range of participants was between 24 and 65 years, and $61 \%$ of them were males. They had hemodialysis duration ranging from 1 to 15 years.

PJ consumption did not affect anthropometric measures including weight, BMI, waist circumference, skeletal muscle and fat mass; no significant difference was either observed between the two conditions (Table 2). However, total body water increased significantly in control condition and the changes were significantly different between the conditions $(P=0.009)$. Mini nutritional assessment significantly decreased in $P J$ condition $(P<0.001)$ and increased in the control $(P=0.003)$; the difference between the two conditions was also significant $(P<0.001)$.

Systolic and diastolic blood pressure significantly decreased in PJ condition $(P<0.001)$. Systolic blood pressure increased significantly in control condition and the difference between PJ and control conditions for both systolic and diastolic blood pressure was significant $(P<0.001)$. Triglycerides decreased in PJ condition $(P<0.001)$ and increased in the control $(P=0.007)$. In contrast, HDL cholesterol increased in PJ condition $(P<0.001)$ and decreased in the control $(P=0.001)$. There was a significant between-condition difference for both triglycerides and HDL cholesterol $(P<0.001)$. Total and LDL cholesterol did not significantly change in either condition. Similarly, no significant alteration in AST and ALT was observed within or between the conditions.

Total antioxidant capacity increased in PJ condition $(P<0.001)$ and decreased in the control $(P<0.001)$. Conversely, malondialdehyde and IL-6 decreased in $\mathrm{PJ}$ condition $(\mathrm{P}<0.001)$ and increased in the control $(P \leq 0.001)$. The changes of these parameters were significantly different between the two conditions.

Serum sodium, calcium, and phosphate did not change significantly during the study in either condition but potassium levels increased in $\mathrm{PJ}$ condition ( $\mathrm{P}=0.01)$ with no significant difference between control and PJ conditions. Dietary intakes did not differ between PJ and control conditions (Table 3). 


\section{Discussion}

Our study showed that 8-week PJ consumption ( $100 \mathrm{ml} /$ day three times a week) may have beneficial effects on blood pressure, serum triglycerides and HDL cholesterol of hemodialysis patients. PJ may also have antioxidant and anti-inflammatory effect as it increased total antioxidant capacity and decreased malondialdehyde and IL-6. In the amounts consumed, PJ did not have adverse effects on potassium levels.

Hypertension is one of medical problems in hemodialysis patients mostly originated from fluid overload [20]. Conversely, reduction of total body water in CKD could be associated with better blood pressure control [23]. In the current study, a significant difference was observed between PJ and control conditions in the amount of total body water. This difference occurred as a result of non-significant decrease in total body water of patients in PJ condition and a significant increase in total body water of patients in the control. These alterations in total body water were concordant with changes in blood pressure in each condition. Although a cause-effect relationship could not be predicated from results presented here, other investigators have shown that reduction in total body water may improve blood pressure in hemodialysis patients [23].

Previous studies have not examined the effect of pomegranate or polyphenols on total body water content of hemodialysis patients but the blood pressure-lowering effect of PJ has been reported in previous trials on both normotensive [24] and hypertensive individuals [25], patients with metabolic syndrome [26], and hemodialysis patients [18]. A meta-analysis of randomized controlled trials confirmed this effect independent of duration or dose of PJ [27]. The beneficial effect of pomegranate on blood pressure may be due to improved nitric oxide bioavailability through scavenging reactive oxygen species [28] as well as enhanced activity and expression of endothelial nitric oxide synthase [29]. In addition, pomegranate may promote vasorelaxation by inhibition of angiotensin converting enzyme [30]. These effects are attributed to phenolic compounds of pomegranate [31].

In contrast to blood pressure, evidence regarding the effect of pomegranate on blood lipids of hemodialysis patients is rather controversial. One study confirmed our results for serum triglycerides and HDL cholesterol [18] but two other investigations reported no effect of either pomegranate polyphenols [16] or PJ [17] on these lipids. Some of the controversy over the results of different studies may be explained by baseline values of triglycerides and HDL cholesterol in participants which likely need to be in unfavorable levels in order for pomegranate to exert its benefit. Also, consumption of pomegranate before dialysis session may reduce its beneficial effect because pomegranate polyphenols pass through dialysis membrane and are discarded in the dialysate [32,33]. Similarly, the lack of PJ effect on total and LDL cholesterol in our study may be partly due to relatively normal levels of these lipids in the participants as other studies on diabetes patients with elevated blood lipids showed promising results $[34,35]$. The lipidlowering and anti-atherogenic properties of pomegranate may relate to a number of mechanisms such as regulating expression of genes involved in lipogenesis and fatty acid oxidation in the liver [36], increasing expression and activity of paraoxonase-1, the antioxidant enzyme in HDL particles that prevents LDL 
oxidation [37], and suppression of oxidized LDL degradation and of cholesterol synthesis in macrophages [38].

In agreement with us, investigations have reported pomegranate antioxidant potential as evidenced by decreased malondialdehyde $[34,35,39,40]$ and increased serum total antioxidant capacity [41]. Likewise, the anti-inflammatory effect of pomegranate has been shown by reduction of $C$ reactive protein $[26,35]$ and IL-6 [35,41], the latter as an inflammatory biomarker with highest predictive value for ESRD outcome [42]. Once again, pomegranate may not exert these effects in non-oxidative stress conditions $[25,43]$ or if administered in pre-dialysis states [17]. Also, such beneficial effects have not been observed in high doses of pomegranate and long intervention lengths [16]. The beneficial effects of pomegranate are attributed to polyphenols [15]. Polyphenols in pomegranate are mostly from anthocyanin subcategory [44] that have great antioxidant and anti-inflammatory properties [15].

Based on the results of MNA, nutritional status was significantly improved in PJ and worsened in control condition. Although less common and valid than subjective global assessment and malnutrition inflammation score methods [45], MNA has been successfully used for assessment of nutritional status in CKD patients [45-48]. A cross-sectional study on hemodialysis patients documented positive correlations between MNA and serum albumin, fat and muscle mass, and BMI and a negative correlation with $C$ reactive protein levels [47]. Nevertheless, no consistency was found in this study between MNA scores and anthropometric characteristics which could be because of relatively short duration of the intervention that did not allow remarkable change in anthropometric measures. In addition, the incongruence between MNA and fat and muscle mass results may be due to low accuracy of BIA in the diagnosis of malnutrition [49].

There were limitations with this study. Due to dietary restrictions and ethnic issues related to hemodialysis patients, as explained in detail in the methods, we could not consider a control treatment for the control arm. Also, BIA may not have shown body composition with sufficient accuracy. More complex techniques such as dual-energy X-ray absorptiometry may determine body composition with more precision. Nonetheless, the study had a crossover design which means that all participants experienced both PJ and control condition, thus reducing risk of selection bias. Also, administration of PJ after dialysis session allows longer cessation of PJ polyphenols in the circulation which enhances their effectiveness. Natural PJ is preferred to pomegranate supplements because it can be embedded in the regular diet more easily.

\section{Conclusions}

In conclusion, post-dialysis consumption of $100 \mathrm{ml} /$ day PJ three times a week for 8 weeks had beneficial effects on blood pressure, serum triglycerides and HDL cholesterol, and oxidative and inflammatory condition of hemodialysis patients without having adverse effect on potassium levels. However, these results may not be generalized to hemodialysis patients from other geographical regions, mainly due to the difference in pomegranate phytochemical composition. 


\section{Abbreviations}

ALT: alanine transaminase; ANCOVA: analysis of covariance; AST: aspartate aminotransferase; BIA: bioelectric impedance analyzer; BMI: body mass index; CKD: chronic kidney disease; ESRD: end-stage renal disease; HDL: high-density lipoprotein; IL-6: interleukin-6; LDL: low-density lipoprotein; SD: standard deviation; MDA: malondialdehyde; MNA: mini nutritional assessment; PJ: pomegranate juice; TAC: total antioxidant capacity; TBARS: thiobarbituric acid reactive substance.

\section{Declarations}

\section{Acknowledgement}

Not applicable.

\section{Funding}

The results presented herein were extracted from the thesis written by Mr. Reza Barati Boldaji. The project was financially supported by Shiraz University of Medical Sciences, grant number. 94-01-84-10361. The funder had no further role in the design of the study and collection, analysis, and interpretation of data or in writing the manuscript.

\section{Availability of data and materials}

The datasets generated during the current study are available from the corresponding author on reasonable request.

\section{Authors' contributions}

RBB and MA contributed to the conception and design of the study. RBB prepared the pomegranate juice and gave it to the participants. RBB and ZE recruited the participants, gave dietary recommendations, conducted the trial, and collected the data. MMS medically supervised the patients. RBB and MA analyzed the data. RBB and ZE drafted the manuscript and MA revised it. All authors read and approved the final version of the manuscript.

\section{Authors' information}


RBB: Department of Community Nutrition, School of Nutrition and Food Sciences, Shiraz University of Medical Sciences, Shiraz, Iran

MA: Nutrition Research Center, Shiraz University of Medical Sciences, Shiraz, Iran

MMS: Department of Nephrology, School of Medicine, Shiraz University of Medical Sciences, Shiraz, Iran

ZE: Department of Clinical Nutrition, School of Nutrition and Food Sciences, Shiraz University of Medical Sciences, Shiraz, Iran

\section{Ethics approval and consent to participate}

All participants gave written informed consent. The study was performed according to guidelines of 1964 Declaration of Helsinki and its later amendments. The trial was approved by the Ethics Committee of Shiraz University of Medical Sciences (approval number: IR.SUMS.REC.1394.210) and registered in the Iranian Registry of Clinical Trials (IRCT 2016070428797N1) at https://en.irct.ir/trial/23270? revision $=23270$.

\section{Consent for publication}

Not applicable.

\section{Competing interests}

The authors declare no conflict of interest.

\section{References}

1. Moradi $H$, Vaziri ND. Molecular mechanisms of disorders of lipid metabolism in chronic kidney disease. Front Biosci (Landmark Ed). 2018 Jan 1;23:146-161.

2. Price AM, Edwards NC, Hayer MK, Moody WE, Steeds RP, Ferro CJ, Townend JN. Chronic kidney disease as a cardiovascular risk factor: lessons from kidney donors. J Am Soc Hypertens. 2018 Jul;12(7):497-505.e4. doi: 10.1016/j.jash.2018.04.010.

3. Ravarotto V, Simioni F, Pagnin E, Davis PA, Calò LA. Oxidative stress - chronic kidney disease cardiovascular disease: a vicious circle. Life Sci. 2018 Oct 1;210:125-131. doi:

10.1016/j.Ifs.2018.08.067.

4. Visconti L, Benvenga S, Lacquaniti A, Cernaro V, Bruzzese A, Conti G, Buemi M, Santoro D. Lipid disorders in patients with renal failure: role in cardiovascular events and progression of chronic kidney disease. J Clin Transl Endocrinol. 2016 Aug 20;6:8-14. doi: 10.1016/j.jcte.2016.08.002. 
5. Qureshi S, Lorch R, Navaneethan SD. Blood pressure parameters and their associations with death in patients with chronic kidney disease. Curr Hypertens Rep. 2017 Oct 18;19(11):92. doi:

10.1007/s11906-017-0790-6.

6. Kooman JP, Dekker MJ, Usvyat LA, Kotanko P, van der Sande FM, Schalkwijk CG, Shiels PG, Stenvinkel P. Inflammation and premature aging in advanced chronic kidney disease. Am J Physiol Renal Physiol. 2017 Oct 1;313(4):F938-F950. doi: 10.1152/ajprenal.00256.2017.

7. Wanner C, Ritz E. Reducing lipids for CV protection in CKD patients-current evidence. Kidney Int Suppl. 2008 Dec;(111):S24-8. doi: 10.1038/ki.2008.520.

8. Gosmanova EO, Kovesdy CP. Blood pressure targets in CKD: lessons learned from sprint and previous observational studies. Curr Cardiol Rep. 2016 Sep;18(9):88. doi: 10.1007/s11886-016-0769y.

9. Daenen K, Andries A4, Mekahli D, Van Schepdael A, Jouret F, Bammens B. Oxidative stress in chronic kidney disease. Pediatr Nephrol. 2018 Aug 13. doi: 10.1007/s00467-018-4005-4.

10. Elsayed ET, Nassra RA, Naga YS. Peroxisome proliferator-activated receptor- $\gamma$-coactivator 1a (PGC1a) gene expression in chronic kidney disease patients on hemodialysis: relation to hemodialysisrelated cardiovascular morbidity and mortality. Int Urol Nephrol. 2017 Oct;49(10):1835-1844. doi: 10.1007/s11255-017-1628-5.

11. Locatelli F, Canaud B, Eckardt KU, Stenvinkel P, Wanner C, Zoccali C. Oxidative stress in end-stage renal disease: an emerging threat to patient outcome. Nephrol Dial Transplant. 2003 Jul;18(7):127280.

12. Liakopoulos V, Roumeliotis S, Gorny X, Dounousi E, Mertens PR. Oxidative stress in hemodialysis patients: a review of the literature. Oxid Med Cell Longev. 2017;2017:3081856. doi:

$10.1155 / 2017 / 3081856$.

13. Tucker PS, Scanlan AT, Dalbo VJ. Chronic kidney disease influences multiple systems: describing the relationship between oxidative stress, inflammation, kidney damage, and concomitant disease. Oxid Med Cell Longev. 2015. Article ID 806358.

14. Viuda-Martos M, Fern'andez-L'opez J, P'erez-'Alvarez JA. Pomegranate and its many functional components as related to human health: a review. Compr Rev Food Sci Food Saf. 2010;9: 645-654.

15. Danesi F, Ferguson LR. Could pomegranate juice help in the control of inflammatory diseases? Nutrients. 2017 Aug 30;9(9). pii: E958. doi: 10.3390/nu9090958.

16. Wu PT, Fitschen PJ, Kistler BM, Jeong JH, Chung HR, Aviram M, Phillips SA, Fernhall B, Wilund KR. Effects of pomegranate extract supplementation on cardiovascular risk factors and physical function in hemodialysis patients. J Med Food. 2015 Sep;18(9):941-9. doi: 10.1089/jmf.2014.0103.

17. Rivara MB, Mehrotra R, Linke L, Ruzinski J, Ikizler TA, Himmelfarb J. A pilot randomized crossover trial assessing the safety and short-term effects of pomegranate supplementation in hemodialysis patients. J Ren Nutr. 2015 Jan;25(1):40-9. doi: 10.1053/j.jrn.2014.07.006.

18. Shema-Didi L, Kristal B, Sela S, Geron R, Ore L. Does pomegranate intake attenuate cardiovascular risk factors in hemodialysis patients? Nutr J. 2014 Mar 4;13:18. doi: 10.1186/1475-2891-13-18. 
19. Shema-Didi L, Sela S, Ore L, Shapiro G, Geron R, Moshe G, Kristal B. One year of pomegranate juice intake decreases oxidative stress, inflammation, and incidence of infections in hemodialysis patients: a randomized placebo-controlled trial. Free Rad Biol Med. 2012;53(2):297-304.

20. Santi Xavier P, Perez Vogt B, Cuadrado Martin L, Vaninni F, Araújo Antunes A, Ponce D, Costa Teixeira Caramori J, Dos Santos E Silva Martin R, da Silva Franco RJ, Barretti P. Total body water and failure to control blood pressure by medication in hemodialysis patients. Nephron Extra. 2014 Jul 3;4(2):95-100. doi: 10.1159/000363322.

21. Satoh K. Serum lipid peroxide in cerebrovascular disorders determined by a new colorimetric method. Clin Chim Acta.1978 Nov 15;90(1):37-43.

22. Asiimwe SB. Simplifications of the mini nutritional assessment short-form are predictive of mortality among hospitalized young and middle-aged adults. Nutrition. 2016 Jan;32(1):95-100. doi: 10.1016/j.nut.2015.07.017.

23. de Castro Júnior JR, Fernandes N, Lacet TB, Maia FS, Bonato GR, Nogueira C, Barberato SH, de Paula RB. Total body water reduction in subjects with chronic kidney disease on peritoneal dialysis is associated with a better hypertension control. J Bras Nefrol. 2014 Oct-Dec;36(4):482-9. doi: 10.5935/0101-2800.20140069.

24. Stockton A, Farhat G, McDougall GJ, Al-Dujaili EAS. Effect of pomegranate extract on blood pressure and anthropometry in adults: a double-blind placebo-controlled randomized clinical trial. J Nutr Sci. 2017 Aug 9;6:e39. doi: 10.1017/jns.2017.36.

25. Asgary S, Sahebkar A, Afshani MR, Keshvari M, Haghjooyjavanmard S, Rafieian-Kopaei M. Clinical evaluation of blood pressure lowering, endothelial function improving, hypolipidemic and antiinflammatory effects of pomegranate juice in hypertensive subjects. Phytother Res. 2014 Feb;28(2):193-9. doi: 10.1002/ptr.4977.

26. Moazzen $\mathrm{H}$, Alizadeh M. Effects of pomegranate juice on cardiovascular risk factors in patients with metabolic syndrome: a double-blinded, randomized crossover controlled trial. Plant Foods Hum Nutr. 2017 Jun;72(2):126-133. doi: 10.1007/s11130-017-0605-6.

27. Sahebkar A, Ferri C, Giorgini P, Bo S, Nachtigal P, Grassi D. Effects of pomegranate juice on blood pressure: A systematic review and meta-analysis of randomized controlled trials. Pharmacol Res. 2017 Jan;115:149-161. doi: 10.1016/j.phrs.2016.11.018.

28. Ignarro LJ, Byrns RE, Sumi D, de Nigris F, Napoli C. Pomegranate juice protects nitric oxide against oxidative destruction and enhances the biological actions of nitric oxide. Nitric Oxide. 2006 Sep;15(2):93-102.

29. Vilahur G, Padró T, Casaní L, Mendieta G, López JA, Streitenberger S, Badimon L. Polyphenolenriched diet prevents coronary endothelial dysfunction by activating the Akt/eNOS pathway. Rev Esp Cardiol (Engl Ed). 2015 Mar;68(3):216-25. doi: 10.1016/j.rec.2014.04.021.

30. Stowe CB. The effects of pomegranate juice consumption on blood pressure and cardiovascular health. Complement Ther Clin Pract. 2011 May;17(2):113-5. doi: 10.1016/j.ctcp.2010.09.004. 
31. Medina-Remón A, Tresserra-Rimbau A, Pons A, Tur JA, Martorell M, Ros E4, Buil-Cosiales P, Sacanella E, Covas MI, Corella D, Salas-Salvadó J, Gómez-Gracia E, Ruiz-Gutiérrez V, Ortega-Calvo M11, GarcíaValdueza M, Arós F, Saez GT, Serra-Majem L, Pinto X, Vinyoles E, Estruch R, Lamuela-Raventos RM; PREDIMED Study Investigators. Effects of total dietary polyphenols on plasma nitric oxide and blood pressure in a high cardiovascular risk cohort. The PREDIMED randomized trial. Nutr Metab Cardiovasc Dis. 2015 Jan;25(1):60-7. doi: 10.1016/j.numecd.2014.09.001.

32. Erben J, Schück O, Brátová M, Moucka P, Palicka V, Fixa P, Hájková B, Herout V, Stilec R, Zahradník $\mathrm{J}$. Kinetics of phenols in body fluid compartments during hemodialysis. Artif Organs. 1995 Jun;19(6):544-6.

33. Nowak PJ, Wilk R, Prymont-Przyminska A, Zwolinska A, Sarniak A, Wlodarczyk A, de Graft-Johnson J, Mamelka B, Zasowska-Nowak A, Bartnicki P, Nowak D, Nowicki M. Hemodialysis decreases the concentration of accumulated plant phenols in the plasma of patients on maintenance dialysis: influence of residual renal function. Ther Apher Dial. 2017 Dec;21(6):572-585. doi: 10.1111/17449987.12586.

34. Parsaeyan N, Mozaffari-Khosravi H, Mozayan MR. Effect of pomegranate juice on paraoxonase enzyme activity in patients with type 2 diabetes. J Diabetes Metab Disord. 2012 Aug 31;11(1):11. doi: 10.1186/2251-6581-11-11.

35. Hosseini B, Saedisomeolia A, Wood LG, Yaseri M, Tavasoli S. Effects of pomegranate extract supplementation on inflammation in overweight and obese individuals: A randomized controlled clinical trial. Complement Ther Clin Pract. 2016 Feb;22:44-50. doi: 10.1016/j.ctcp.2015.12.003.

36. Peluso MR. Flavonoids attenuate cardiovascular disease, inhibit phosphodiesterase, and modulate lipid homeostasis in adipose tissue and liver. Exp Biol Med (Maywood). 2006 Sep;231(8):1287-99.

37. Estrada-Luna D, Martínez-Hinojosa E, Cancino-Diaz JC, Belefant-Miller H, López-Rodríguez G, Betanzos-Cabrera G. Daily supplementation with fresh pomegranate juice increases paraoxonase 1 expression and activity in mice fed a high-fat diet. Eur J Nutr. 2018 Feb;57(1):383-389. doi: 10.1007/s00394-017-1394-2.

38. Fuhrman B, Volkova N, Aviram M. Pomegranate juice inhibits oxidized LDL uptake and cholesterol biosynthesis in macrophages. J Nutr Biochem. 2005 Sep;16(9):570-6.

39. Kojadinovic MI, Arsic AC, Debeljak-Martacic JD, Konic-Ristic Al, Kardum ND, Popovic TB, Glibetic MD. Consumption of pomegranate juice decreases blood lipid peroxidation and levels of arachidonic acid in women with metabolic syndrome. J Sci Food Agric. 2017 Apr;97(6):1798-1804. doi:

10.1002/jsfa.7977.

40. Razani Z, Dastani M, Kazerani HR. Cardioprotective effects of pomegranate (punica granatum) juice in patients with ischemic heart disease. Phytother Res. 2017 Nov;31(11):1731-1738. doi: 10.1002/ptr.5901.

41. Shishehbor F, Mohammad Shahi M, Zarei M, Saki A, Zakerkish M, Shirani F, Zare M. Effects of concentrated pomegranate juice on subclinical inflammation and cardiometabolic risk factors for 
type 2 diabetes: a quasi-experimental study. Int J Endocrinol Metab. 2016 Jan 30;14(1):e33835. doi: 10.5812/ijem.33835.

42. Elewa U, Sanchez-Niño MD, Martin-Cleary C, Fernandez-Fernandez B, Egido J, Ortiz A. Cardiovascular risk biomarkers in CKD: the inflammation link and the road less traveled. Int Urol Nephrol. 2012 Dec;44(6):1731-44. doi: 10.1007/s11255-012-0271-4.

43. Manthou E, Georgakouli K, Deli CK, Sotiropoulos A, Fatouros IG, Kouretas D, Haroutounian S, Matthaiou C, Koutedakis Y, Jamurtas AZ. Effect of pomegranate juice consumption on biochemical parameters and complete blood count. Exp Ther Med. 2017 Aug;14(2):1756-1762. doi: 10.3892/etm.2017.4690.

44. Kalaycıoğlu Z, Erim FB. Total phenolic contents, antioxidant activities, and bioactive ingredients of juices from pomegranate cultivars worldwide. Food Chem. 2017 Apr 15;221:496-507. doi: 10.1016/j.foodchem.2016.10.084.

45. Santin FG, Bigogno FG, Dias Rodrigues JC, Cuppari L, Avesani CM. Concurrent and predictive validity of composite methods to assess nutritional status in older adults on hemodialysis. J Ren Nutr. 2016 Jan;26(1):18-25. doi: 10.1053/j.jrn.2015.07.002.

46. Rogowski Ł, Kusztal M, Gołębiowski T, Bulińska K, ZembrońŁacny A, Wyka J, Klinger M, Woźniewski M, Dziubek W. Nutritional assessment of patients with end-stage renal disease using the MNA scale. Adv Clin Exp Med. 2018 Aug;27(8):1117-1123. doi: 10.17219/acem/75604.

47. Erdoğan E, Tutal E, Uyar ME, Bal Z, Demirci BG, Sayın B, Sezer S. Reliability of bioelectrical impedance analysis in the evaluation of the nutritional status of hemodialysis patients - a comparison with Mini Nutritional Assessment. Transplant Proc. 2013;45(10):3485-8. doi: 10.1016/j.transproceed.2013.08.096.

48. Tsai HJ, Tsai AC, Hung SY, Chang MY. Comparing the predictive ability of population-specific MiniNutritional Assessment with Subjective Global Assessment for Taiwanese patients with hemodialysis: a cross-sectional study. Int J Nurs Stud. 2011 Mar;48(3):326-32. doi: 10.1016/j.jnurstu.2010.07.015.

49. da Silva AT, Hauschild DB, Moreno YMF, Bastos JLD, Wazlawik E. Diagnostic accuracy of bioelectrical impedance analysis parameters for the evaluation of malnutrition in patients receiving hemodialysis. Nutr Clin Pract. 2018 May 22. doi: 10.1002/ncp.10098. [Epub ahead of print]

\section{Tables}

Table 1 - Baseline characteristics of the participants 


\begin{tabular}{lc}
\hline Characteristics & Mean \pm SD or $\mathbf{n}(\%)$ \\
\hline Age $(\mathrm{y})$ & $47.8 \pm 13.3$ \\
Males $(\mathrm{n})$ & $25(61.0)$ \\
\hline Weight $(\mathrm{kg})$ & $65.7 \pm 15.5$ \\
\hline Body mass index $\left(\mathrm{kg} / \mathrm{m}^{2}\right)$ & $23.9 \pm 4.8$ \\
\hline Mini nutritional assessment (points) & $15.2 \pm 2.8$ \\
\hline Systolic blood pressure $(\mathrm{mm} \mathrm{Hg})$ & $135.9 \pm 8.3$ \\
\hline Diastolic blood pressure $(\mathrm{mm} \mathrm{Hg})$ & $98.0 \pm 9.5$ \\
\hline Triglycerides $(\mathrm{mg} / \mathrm{dl})$ & $154.4 \pm 75.1$ \\
\hline Total cholesterol $(\mathrm{mg} / \mathrm{dl})$ & $150.1 \pm 38.0$ \\
\hline LDL cholesterol $(\mathrm{mg} / \mathrm{dl})$ & $76.1 \pm 25.7$ \\
\hline HDL cholesterol $(\mathrm{mg} / \mathrm{dl})$ & $43.3 \pm 10.3$ \\
\hline AST $(\mathrm{U} / \mathrm{L})$ & $13.6 \pm 7.8$ \\
\hline ALT $(\mathrm{U} / \mathrm{L})$ & $11.1 \pm 7.1$ \\
\hline
\end{tabular}

Data are expressed as either mean \pm standard deviation (SD) or number and percentage. $\mathrm{n}=41$. Abbreviations: ALT, alanine aminotransferase; AST, aspartate aminotransferase; HDL, high-density lipoprotein; LDL, low-density lipoprotein.

Table 2 - The effect of 8 weeks' PJ consumption on anthropometric, blood pressure, and biochemical measurements (values are the sum of both sequences) $\square$ 


\section{Baseline}

Week 8
Difference (95\% CI)
$P$ value value (treatment $x$ $(\text { time })^{2}$ time) $)^{3}$
Weight (kg)

PJ condition

Control condition
$65.65 \pm 15.67 \quad 65.78 \pm 15.41$

$65.65 \pm 15.67 \quad 65.65 \pm 15.45$
$0.13(-0.11,0.38)$

$0.00(-0.22,0.22)$

Body mass index $\left(\mathrm{kg} / \mathrm{m}^{2}\right)$

PJ condition

Control condition
$23.88 \pm 4.86$

$23.88 \pm 4.86$
$23.93 \pm 4.77$

$23.88 \pm 4.77$
$0.05(-0.03,0.14)$

$0.00(-0.08,0.08)$
0.27

0.98

0.23

1

0.24

Waist circumference (cm)
PJ condition
$85.63 \pm 13.5086 .17 \pm 14.10$
$0.54(-0.33,1.41)$
0.22
Control condition
$84.18 \pm 15.1183 .85 \pm 14.88$
$-0.33(-1.10,0.45)$
0.40
0.14

Fat mass (kg)

PJ condition

Control condition
$17.81 \pm 11.7618 .02 \pm 11.73$

$18.35 \pm 12.1617 .21 \pm 11.84$
$0.21(-0.19,0.61)$

$-1.15(-2.76,0.47)$

0.29

0.16

0.10

Skeletal muscle mass (kg)

PJ condition

Control condition

Total body water (kg)

PJ condition

Control condition

$$
26.73 \pm 5.85
$$

$26.67 \pm 6.12$

$$
26.58 \pm 5.63
$$

$26.88 \pm 6.00$
$-0.14(-0.43,0.14)$

$0.21(-0.13,0.55)$
0.32

0.21

0.10
$35.83 \pm 7.21$

$35.72 \pm 7.49$
$35.62 \pm 6.97$

$36.11 \pm 7.07$
$-0.21(-0.53,0.11)$

$0.38(0.03,0.74)$
0.19

0.04

0.008

Mini nutritional assessment

(points)

PJ condition

Control condition

Systolic blood pressure ( $\mathrm{mm} \mathrm{Hg}$ )

PJ condition

Control condition

Diastolic blood pressure ( $\mathrm{mm} \mathrm{Hg}$ )

PJ condition

Control condition

Triglycerides (mg/dL)

$$
\text { PJ condition }
$$

Control condition

Total cholesterol (mg/dL)

$$
\text { PJ condition }
$$

Control condition

$\begin{array}{ll}15.68 \pm 2.79 & 14.18 \pm 2.65 \\ 14.88 \pm 2.88 & 15.50 \pm 2.65\end{array}$

$-1.50(-1$.

$0.63(0.23,1.02)$

$<0.001$

0.003 $<0.001$

$135.65 \pm 8.47 \quad 128.69 \pm 6.11$ $135.95 \pm 8.27136 .93 \pm 7.46$

$-6.97(-4.88,-9.05)<0.001$

$0.97(0.11,1.84)$

0.03 $<0.001$

\section{$97.73 \pm 9.95$}

$90.84 \pm 6.86$

$-6.88(-9.08,-4.68)$

$<0.001$

$98.20 \pm 9.34$

$99.18 \pm 8.88$

$0.98(-0.29,2.24)$

0.13

$<0.001$

$157.22 \pm$

69.79

$$
\begin{gathered}
150.77 \pm \\
81.59
\end{gathered}
$$

$125.32 \pm 58.24$

$171.67 \pm$ 102.59

$$
\begin{aligned}
& -31.90(-18.77, \quad<0.001 \\
& -45.04)
\end{aligned}
$$

$20.90(6.16,35.64) \quad 0.007$

$<0.001$

$$
155.07 \pm \quad 149.82 \pm 36.46-5.26(-16.96,6.44) \quad 0.37
$$
38.97

$$
\begin{array}{lllll}
144.25 \pm & 145.85 \pm 30.45 & 1.60(-6.87,10.07) & 0.70 & 0.73 \\
36.80 & & & &
\end{array}
$$

LDL cholesterol (mg/dL)

PJ condition

Control condition
$80.20 \pm 25.9677 .77 \pm 23.86$

$71.82 \pm 25.3875 .80 \pm 28.60$
$-2.43(-9.16,4.30)$

$3.97(-1.92,9.87)$
0.47

0.18
0.34

HDL cholesterol (mg/dL) 
PJ condition

Control condition

AST (U/L)

PJ condition

Control condition

ALT (U/L)

PJ condition

Control condition

Total antioxidant capacity

$(\mathrm{mmol} / \mathrm{L})$

PJ condition
Control condition
Malondialdehyde $(\mu \mathrm{mol} / \mathrm{L})$

PJ condition

Control condition

Interleukin-6 (ng/L)

PJ condition

Control condition

Sodium (mEq/L)

PJ condition

Control condition

Potassium (mEq/L)

PJ condition
Control condition
alcium $(\mathrm{mg} / \mathrm{dL})$

PJ condition

$7.95 \pm 0.41$

Control condition

$5.56 \pm 0.28 \quad 5.69 \pm 0.30$

$5.54 \pm 0.25$

$5.57 \pm 0.30$

$0.03(-0.08,1.37)$

$0.03(-0.08,1.37)$

$0.73(-1.90,3.37)$

$1.32(-1.39,4.04)$

0.57

0.33

0.78

0.11 $<0.001$

$8.05 \pm 0.47$

8.97

$7.97 \pm 0.50$

$0.02(-0.20,0.25)$

$0.02(-0.22,0.26)$

$.07 \pm 0.58$

0.83

0.88

0.78

Phosphate (mg/dL)

PJ condition

Control condition

$4.90 \pm 0.45$

$4.93 \pm 0.44$

$5.00 \pm 0.38$

$4.92 \pm 0.40$

$\begin{array}{ll}0.03(-0.16,0.23) & 0.75 \\ 0.08(-0.09,0.26) & 0.33\end{array}$

${ }^{1}$ Data are means \pm SD. $\mathrm{n}=41$ in PJ condition and $\mathrm{n}=40$ in control condition. ${ }^{2} \mathrm{P}$ value assessed by paired samples $t$ test. ${ }^{3}$ Comparisons between the two conditions were performed by ANCOVA (treatment $\times$ time interaction) with age, gender, and baseline values as the covariate. Abbreviations: ALT, alanine aminotransferase; AST, aspartate aminotransferase; HDL, high-density lipoprotein; ITT, intention-to-treat analysis; LDL, low-density lipoprotein.

\section{Table 3 - Dietary intakes during the study periods $\square$}




\begin{tabular}{lccc}
\hline & PJ condition & Control condition P value $^{2}$ \\
\hline Energy (kcal/day) & $1394 \pm 366.5$ & $1310 \pm 328.0$ & 0.30 \\
Carbohydrate (g/day) & $203.8 \pm 60.25$ & $187.5 \pm 51.98$ & 0.21 \\
\hline Protein (g/day) & $58.49 \pm 16.29$ & $56.32 \pm 12.64$ & 0.52 \\
\hline Fat (g/day) & $37.89 \pm 9.73$ & $36.92 \pm 9.98$ & 0.67 \\
\hline Saturated fatty acids (g/day) & $13 . .78 \pm 4.43$ & $13.93 \pm 5.34$ & 0.90 \\
\hline Monounsaturated fatty acids (g/day) & $12.61 \pm 3.05$ & $12.24 \pm 2.94$ & 0.59 \\
\hline Polyunsaturated fatty acids (g/day) & $7.23 \pm 2.09$ & $6.85 \pm 1.60$ & 0.37 \\
\hline Cholesterol (g/day) & $218.57 \pm 76.39$ & $226.14 \pm 69.09$ & 0.65 \\
\hline Fiber (g/day) & $8.54 \pm 3.27$ & $7.43 \pm 2.99$ & 0.13 \\
\hline Vitamin E (mg/day) & $2.25 \pm 0.93$ & $2.24 \pm 1.17$ & 0.94 \\
\hline Vitamin C (mg/day) & $35.30 \pm 16.24$ & $31.67 \pm 10.68$ & 0.26 \\
\hline Carotenoids ( $\mu$ g/day) & $409.0 \pm 542.1$ & $524.3 \pm 517.0$ & 0.35 \\
\hline Sodium (mg/day) & $1475 \pm 517.5$ & $1452 \pm 565.6$ & 0.86 \\
\hline Potassium (mg/day) & $1486 \pm 399.2$ & $1361 \pm 320.8$ & 0.14 \\
\hline Calcium (mg/day) & $574.2 \pm 168.5$ & $620.4 \pm 198.3$ & 0.28 \\
\hline Phosphorus (mg/day) & $796.7 \pm 189.2$ & $780.7 \pm 173.9$ & 0.70 \\
\hline
\end{tabular}

${ }^{1}$ Data are means \pm SD. $n=41$ in PJ condition and $n=40$ in control condition. ${ }^{2} \mathrm{P}$ value assessed by independent samples t test. ITT, intention-to-treat analysis.

\section{Figures}

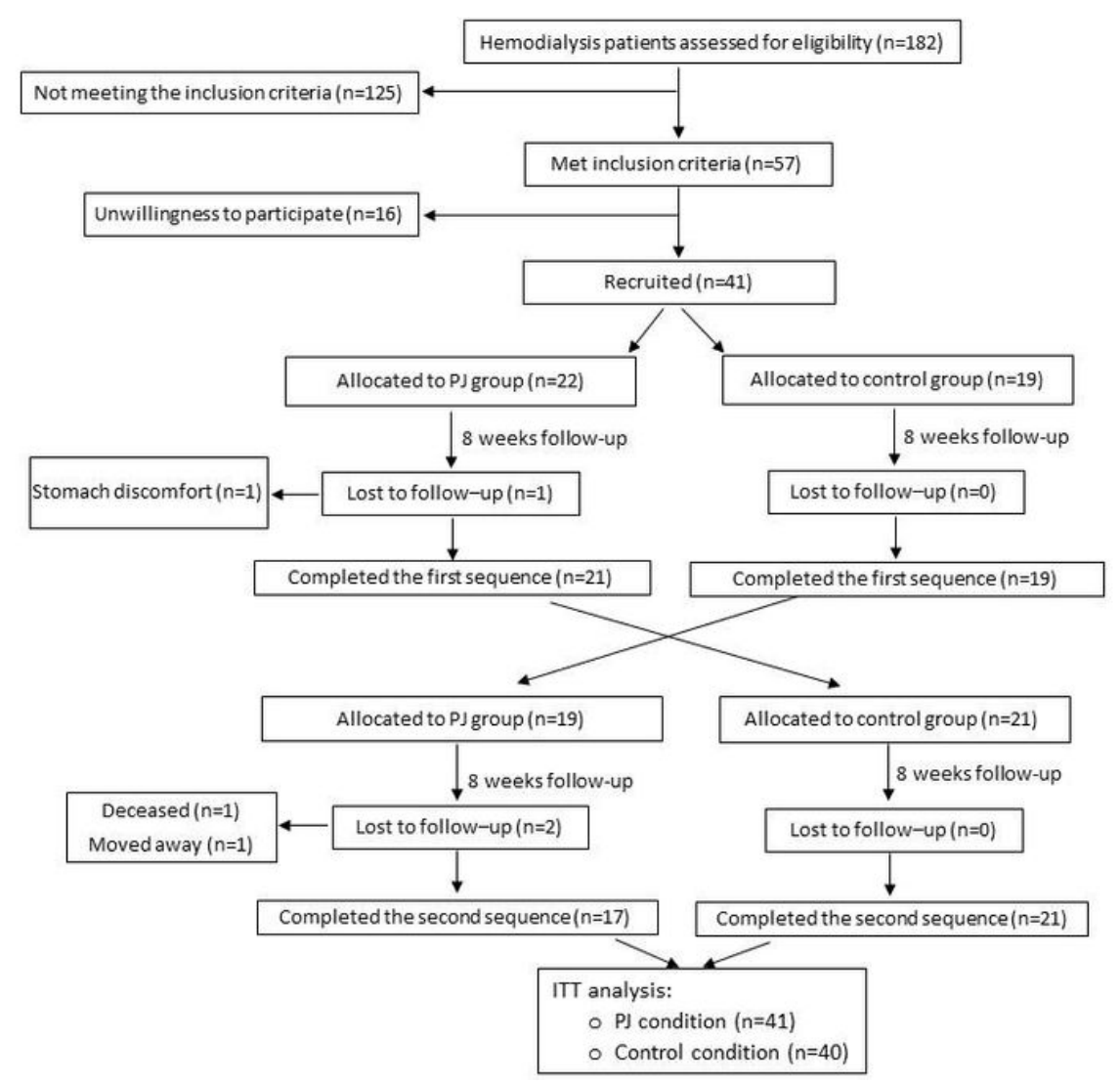

Figure 1

Page 18/19 
Flowchart of the trial - ITT, intention-to-treat.

\section{Supplementary Files}

This is a list of supplementary files associated with this preprint. Click to download.

- supplement1.doc 